\title{
Controversies and consensus regarding vitamin D deficiency in 2015: whom to test and whom to treat?
}

\section{Controversy persists regarding who should be tested and who should be treated for vitamin $\mathrm{D}$ deficiency}

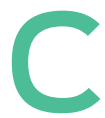

ontroversy continues to surround vitamin D testing, the diagnosis and clinical significance of vitamin D deficiency, and the benefits - or lack thereof - of vitamin D supplementation. Over 2000 peer-reviewed articles have been published on these topics within the past 12 months (at time of writing), generating much debate and discussion in the scientific literature and lay media.

\section{The role of vitamin D in skeletal and extraskeletal health}

Vitamin D has an established and important role in skeletal health through its actions in mediating intestinal calcium absorption and, therefore, its effects on extracellular calcium homeostasis and bone mineralisation. Consequently, severe vitamin $\mathrm{D}$ deficiency results in under-mineralisation of bone (osteomalacia in adults, rickets in children) that requires treatment with vitamin $\mathrm{D}$ and calcium supplementation. Vitamin D deficiency is also associated with secondary hyperparathyroidism and increased bone turnover, which may contribute to osteoporosis and fracture risk.

Since the publication of a landmark randomised, placebo-controlled trial that showed that vitamin D (cholecalciferol) and calcium supplementation reduced hip and non-vertebral fractures in a group of elderly, vitamin D-deficient women, ${ }^{1}$ the correction of vitamin $\mathrm{D}$ deficiency and assurance of adequate calcium intake have been cornerstones of osteoporosis management. Indeed, most of the evidence for the fracture reduction efficacy of the antiresorptive therapies currently prescribed has been from clinical trials in which the study participants were vitamin D- and calciumreplete or supplemented as needed.

However, whether vitamin D and calcium supplementation is required in the majority of patients treated for osteoporosis has been challenged. Some studies have suggested that the efficacy of antiresorptive therapies is independent of baseline vitamin D status or calcium intake, ${ }^{2}$ but these conclusions were based, in part, on post-hoc secondary analyses in people who, in the majority, did not have vitamin $\mathrm{D}$ deficiency, using bone density as the primary end point. Moreover, a recent trial sequential meta-analysis demonstrated that vitamin D supplementation alone did not reduce total or hip fracture risk, but co-supplementation with vitamin D and calcium did, with sensitivity analyses suggesting that elderly institutionalised patients might benefit the most. ${ }^{3}$ Therefore, while it is unclear whether serum vitamin D concentration itself is a useful marker of osteoporosis risk, ${ }^{4}$ and vitamin D supplementation alone may not improve skeletal outcomes in many patients at risk of osteoporosis who might be otherwise calcium sufficient, there is evidence that older individuals at increased risk of vitamin D deficiency should be targeted for supplementation with both vitamin $\mathrm{D}$ and calcium to reduce fracture risk.

Even more controversial is the role of vitamin D in extraskeletal diseases such as cancer, atherosclerosis, diabetes, infections and neurodegenerative diseases. Vitamin D receptors are expressed in many non-skeletal tissues where conversion of 25-hydroxyvitamin D $(25(\mathrm{OH}) \mathrm{D})$ to active metabolites through local enzymatic action can potentially result in a wide variety of paracrine or autocrine effects, ${ }^{5}$ ranging from antiproliferation to immunomodulation. Numerous epidemiological studies show an inverse relationship between $25(\mathrm{OH}) \mathrm{D}$ concentrations and a wide range of illnesses, but these observational data are limited by possible reverse causation, residual confounding, and classification and publication biases. ${ }^{6}$ Illness can result in the contraction of outdoor activities, reduced sunlight exposure and, accordingly, low $25(\mathrm{OH}) \mathrm{D}$ concentration may be a consequence, rather than a cause, of disease.

\section{Low vitamin D may be a marker of ill health}

To minimise the impact of reverse causation, a recent systematic review examining the relationship between $25(\mathrm{OH}) \mathrm{D}$ concentration and ill health analysed prospective and nested case-control studies where the disorder of interest was not previously diagnosed and only studies that measured $25(\mathrm{OH}) \mathrm{D}$ concentrations, rather than predicted vitamin $\mathrm{D}$ status according to sunlight exposure or dietary intake, were included, to limit classification bias. ${ }^{7}$ The authors confirmed that most prospective observational studies showed an inverse association between $25(\mathrm{OH}) \mathrm{D}$ concentrations and a number of diverse health outcomes. However, to minimise residual confounding and determine causality, analysis of the randomised trials of vitamin D supplementation was also undertaken. This showed, almost universally, that vitamin D supplementation had little or no effect on the occurrence, severity and clinical course of these illnesses - even after subgroup analyses of subjects with vitamin D deficiency who received adequate dose supplementation. The discrepancy between the observational and interventional trial findings suggests that low 25(OH)D doi: 10.5694/mja14.00702 may be a marker, rather than a cause, of ill health 
- perhaps reflecting the effects of inflammation and the negative acute phase response of vitamin D-binding protein.

Interestingly, among the interventional trial data, a small survival benefit was seen in a subgroup of frail older women, a sizeable proportion of whom were in institutional care. This again suggests that frail older patients at increased risk of significant vitamin $\mathrm{D}$ deficiency might benefit from supplementation. Vitamin D supplementation in older patients - using cholecalciferol but not ergocalciferol - was also found to be associated with a modest reduction in overall mortality in another recently published meta-analysis. ${ }^{8}$

\section{Benefits of vitamin D supplementation may} not exceed the costs of unnecessary testing

Taken together, the current evidence suggests that the main beneficial effects of vitamin D supplementation relate to musculoskeletal, rather than extraskeletal, health outcomes, with the subset of frail older patients with the highest likelihood of vitamin D deficiency being those most likely to benefit. Nonetheless, the exponential increase in vitamin $\mathrm{D}$ testing and supplement use in recent years, not just in Australia but worldwide, has raised justifiable concerns that many vitamin $\mathrm{D}$ measurements are being undertaken without evidence-supported indications ${ }^{6}$ and many individuals are being supplemented with little evidence for benefit. Australian Medicare billing data have shown a remarkable 94 -fold increase in vitamin D testing between 2000 and 2010, with repeat testing accounting for nearly half the test numbers, despite only a 0.5 -fold increase in bone mineral density testing over the same period. ${ }^{9}$

\section{Royal College of Pathologists of Australasia} position statement on vitamin D testing

In response to these concerns about possible inappropriate "over-testing", the Royal College of Pathologists of Australasia (RCPA) convened a working party and, in 2013, published a position statement to clarify the role of vitamin D testing in the context of vitamin $\mathrm{D}$ deficiency, with guidelines about who should be tested and when repeat testing should be performed. ${ }^{10}$

The RCPA recommendations, broadly consistent with the current evidence, advocate selective testing as an appropriate case-finding strategy in individuals at increased risk of vitamin D deficiency, and their suggested clinical indications for vitamin D measurement support this conclusion. Specifically, the routine screening of healthy adults is not recommended, with the caution that doing so might reveal a significantly sizeable group with low vitamin $\mathrm{D}$ levels that could lead to treatment without clear evidence of benefit and perpetuate unnecessary repeat testing. The statement also affirms the use of
$25(\mathrm{OH}) \mathrm{D}$ as the best marker of vitamin $\mathrm{D}$ status but acknowledges that variability exists between the current assay methods. Progress is presently being made to overcome some of these methodological limitations (eg, interference from heterophilic antibodies, inefficient separation of analyte from binding protein) and improve standardisation, through the use of international serum-based reference standards, the adoption of a reference method and the introduction of an international vitamin D certification program administered by the US Centers for Disease Control and Prevention.

The recommended 25(OH)D target treatment threshold of $50 \mathrm{nmol} / \mathrm{L}$ (at the end of winter), supported by Australian $^{5}$ and international guidelines, is based on skeletal health outcomes and surrogate end points. The RCPA position statement emphasises the current lack of consistent evidence for the benefit of vitamin D supplementation in the treatment and prevention of many extraskeletal illnesses. Based on the serum half-life of $25(\mathrm{OH}) \mathrm{D}$ and basic pharmacokinetic principles, repeat vitamin D testing should occur no earlier than 3 months after the commencement of supplementation or a change in dose, and no further testing may be required once the target $25(\mathrm{OH}) \mathrm{D}$ concentration is achieved.

In the climate of rising costs and limited resources, it behoves clinicians to ensure that their requests for vitamin D measurement and their prescription of vitamin D supplements are evidence-based, or at least supported by established guidelines and expert consensus, to avoid unnecessary and inappropriate testing, the medicalisation of otherwise healthy lowrisk individuals and the treatment of patients who will not clearly benefit from supplementation.

The rationalisation of vitamin D measurement could foreseeably begin with education initiatives to increase general awareness and appreciation of the current evidence and guidelines for appropriate testing and supplementation. This could be followed by audit of local practice to provide data and feedback to improve and streamline guideline- and evidence-supported test requests and subsequent management. The latter initiatives may be easier to tackle than imposing limitations on indications for vitamin D testing, as announced by Medicare Australia after review by the Medical Services Advisory Committee in 2014. The results of ongoing large prospective randomised clinical trials, ${ }^{11}$ such as the current Australian D-Health trial (http://dhealth.qimrberghofer.edu.au), will hopefully further clarify the role of vitamin D supplementation in the prevention and management of skeletal and non-skeletal disorders, including its effects on mortality risk, in various patient and individual subgroups.

Competing interests: No relevant disclosures.

Provenance: Not commissioned; externally peer reviewed.

References are available online at www.mja.com.au. 
1 Chapuy MC, Arlot ME, Duboeuf F, et al. Vitamin D3 and calcium to prevent hip fractures in the elderly women. $\mathrm{NEngl}$ J Med 1992; 327: 1637-1642.

2 Bourke S, Bolland MJ, Grey A, et al. The impact of dietary calcium intake and vitamin D status on the effects of zoledronate. Osteoporos Int 2013; 24: 349-354.

3 Bolland MJ, Grey A, Gamble GD, Reid IR. The effect of vitamin D supplementation on skeletal, vascular, or cancer outcomes: a trial sequential meta-analysis. Lancet Diabetes Endocrinol 2014; 2: 307-320.

4 Rosen CJ, Taylor CL. Common misconceptions about vitamin D - implications for clinicians. Nat Rev Endocrinol 2013; 9: 434-438.

5 Nowson CA, McGrath JJ, Ebeling PR, et al. Vitamin D and health in adults in Australia and New Zealand: a position statement. Med J Aust 2012; 196: 686-687.

6 Sattar N, Welsh P, Panarelli M, Forouhi NG. Increasing requests for vitamin $D$ measurement: costly, confusing, and without credibility. Lancet 2012; 379: 95-96.
7 Autier P, Boniol M, Pizot C, Mullie P. Vitamin D status and ill health: a systematic review. Lancet Diabetes Endocrinol 2014; 2: 76-89.

8 Chowdhury R, Kunutsor S, Vitezova A, et al. Vitamin D and risk of cause specific death: systematic review and meta-analysis of observational cohort and randomised intervention studies. BMJ 2014; 348: g1903. doi: 10.1136/bmj.g1903.

9 Bilinski K, Boyages S. Evidence of overtesting for vitamin D in Australia: an analysis of 4.5 years of Medicare Benefits Schedule (MBS) data. BMJ Open 2013; 3: pii: e002955.

10 Royal College of Pathologists of Australasia. Position statement: use and interpretation of vitamin D testing. Number: 1/2013. May 2013. http://www.rcpa.edu.au/ Library/College-Policies/Position-Statements/Use-andInterpretation-of-Vitamin-D-Testing (accessed Apr 2015).

1 Kupferschmidt K. Uncertain verdict as vitamin $D$ goes on trial. Science 2012; 337: 1476-1478. 